\title{
The Monoid Rank and Monoid Presentation of Order-Preserving and Order-Decreasing Full Contraction Mappings
}

\author{
Kemal Toker*
}

\begin{abstract}
Let $n \in \mathbb{Z}^{+}$and $X_{n}=\{1,2, \ldots, n\}$ be a finite set. Let $\mathcal{O} D C T_{n}$ be the order-preserving and orderdecreasing full contraction mappings on $X_{n}$. It is well known that $\mathcal{O} D C T_{n}$ is a monoid. In this paper, we have found the monoid rank and monoid presentation of $\mathcal{O} D C T_{n}$. In particular, we have proved that monoid rank of $\mathcal{O D C T} T_{n}$ is $n-1$ for $n \in \mathbb{Z}^{+}$and $<a_{1}, a_{2}, \ldots, a_{n-1} \mid a_{i} a_{n-1}=a_{i}(1 \leq i \leq n-1), a_{i} a_{j}=$ $a_{j+1} a_{i}(1 \leq i \leq j \leq n-2)>$ is a monoid presentation of $\mathcal{O} D C T_{n}$ for $n \geq 3$.
\end{abstract}

Keywords: Contraction mappings; Generating set; Monoid presentation.

AMS Subject Classification (2020): 20M20.

${ }^{*}$ Corresponding author

\section{Introduction}

Let $X$ be a non-empty set and let $\mathcal{T}_{X}$ be the full transformation semigroup on $X$. Every semigroup is isomorphic to a subsemigroup of full transformation semigroup [7]. So, the full transformation semigroup is ubiquitous in the semigroup theory. Let $n \in \mathbb{Z}^{+}$and $X_{n}=\{1,2, \ldots, n\}$ be a finite set. We use $\mathcal{T}_{n}$ instead of $\mathcal{T}_{X_{n}}$ for convenience.

Let $M$ be a monoid and A be any subset of $M$. Then the submonoid of $M$ by generated $A$ (which is the smallest submonoid of $M$ containing $A$ ) is denoted by $\langle A\rangle$. If $\langle A\rangle=M$ while the cardinality of $A$ is a finite number, then $M$ is called finitely generated monoid. With a similar idea, by replacing $M$ by a semigroup $S$, one may define finitely generated semigroup as well.

The monoid rank of finitely generated monoid $M$ is defined by

$$
\operatorname{rank}_{M}(M)=\min \{|A|:<A>=M\} .
$$

Let $\mathcal{C} T_{n}$ be the full contraction transformations on $X_{n}$, it is defined by

$$
\mathcal{C} T_{n}=\left\{\alpha \in \mathcal{T}_{n}\left|\left(\forall x, y \in X_{n}\right)\right| x \alpha-y \alpha \mid \leq x-y\right\}
$$


and $\mathcal{C} T_{n}$ is a submonoid of $\mathcal{T}_{n}$. Let $\mathcal{O}_{n}$ be the order-preserving full transformations on $X_{n}$ and it is defined by

$$
\mathcal{O}_{n}=\left\{\alpha \in \mathcal{T}_{n} \mid\left(\forall x, y \in X_{n}\right) x \leq y \Longrightarrow x \alpha \leq y \alpha\right\} .
$$

Let $\mathcal{S}_{n}$ be the symmetric group on $X_{n}$. Gomes and Howie have found the semigroup rank of $\mathcal{O}_{n} \backslash \mathcal{S}_{n}=\mathcal{O}_{n} \backslash\left\{1_{S}\right\}$ where $1_{S}$ is the identity mapping of $\mathcal{S}_{n}$ [5]. Let $\mathcal{C}_{n}$ be the order-preserving and order-decreasing transformations on $X_{n}$, it is called Catalan monoid on $X_{n}$ and it is defined by

$$
\mathcal{C}_{n}=\left\{\alpha \in \mathcal{O}_{n} \mid\left(\forall x \in X_{n}\right) x \alpha \leq x\right\}
$$

There are some papers about $\mathcal{C}_{n}$, in the literature such as $[2,6]$. Adeshola and Umar defined a semigroup which is $\mathcal{O}_{n} \cap \mathcal{C} T_{n}$ and they used $\mathcal{O} C T_{n}$ instead of $\mathcal{O}_{n} \cap \mathcal{C} T_{n}$. The cardinalities of some equivalences on $\mathcal{O} C T_{n}$ has been investigated by Adeshola and Umar [1]. Let

$$
\mathcal{D}_{n}=\left\{\alpha \in \mathcal{T}_{n} \mid\left(\forall x \in X_{n}\right) x \alpha \leq x\right\}
$$

be the subsemigroup of $\mathcal{T}_{n}$ consisting of all order-decreasing transformations of $X_{n}$. Moreover, Adeshola and Umar defined a semigroup which is $\mathcal{O} C T_{n} \cap \mathcal{D}_{n}$ and they used $\mathcal{O} D C T_{n}$ instead of $\mathcal{O} C T_{n} \cap \mathcal{D}_{n}$ [1]. $\mathcal{O} D C T_{n}$ is called order-preserving and order-decreasing full contraction mappings. Also, $\mathcal{O} D C T_{n}=\mathcal{C} T_{n} \cap \mathcal{C}_{n}$ thus $\mathcal{O} D C T_{n}$ is a submonoid of $\mathcal{O} C T_{n}$ and submonoid of $\mathcal{C}_{n}$.

Let $A$ be a set, then we denote by $A^{*}$ the free monoid on $A$. Let $R \subseteq A^{*} \times A^{*}$ is a set of pairs of words. An element $(r, s)$ of $R$ is called a relation, and is usually written $r=s$ instead of $(r, s)$. Monoid presentation is an ordered pair $<A \mid R>$ which is the quotient monoid $A^{*} / R^{\#}$ where $R^{\#}$ is the smallest congruence on $A^{*}$ containing $R$. Let $M$ be the monoid defined by $\langle A \mid R\rangle$. Let $w_{1}, w_{2} \in A^{*}$, if $w_{1}$ and $w_{2}$ are identical words on $A^{*}$ then we write $w_{1} \equiv w_{2}$, and we write $w_{1}=w_{2}$ if they represent the same element of the monoid $M$, that is $\left(w_{1}, w_{2}\right) \in R^{\#}$. If $u_{1} \equiv x r y$ and $u_{2} \equiv x s y$ where $x, y \in A^{*}$ and $(r, s) \in R$ or $(s, r) \in R$ then, we say $u_{2}$ is obtained from $u_{1}$ by an application of one relation from $R$. We say that $w_{1}=w_{2}$ is a consequence of $R$, if $w_{1}$ and $w_{2}$ are identical words or if there exists a sequence $w_{1} \equiv u_{1} \rightarrow u_{2} \rightarrow \ldots \rightarrow u_{k} \equiv w_{2}$ where each $u_{i+1}$ is obtained from $u_{i}(1 \leq i \leq k-1)$ by an application of one relation from $R$. Let $T$ be any monoid, let $B$ be a generating set for $T$, and let $\phi: A \rightarrow B$ be an onto mapping. $\phi$ can be extended in a unique way $\bar{\phi}: A^{*} \rightarrow T$. The monoid $T$ is said to satisfy relations $R$ if for each $(u, v) \in R$ we have $u \bar{\phi}=v \bar{\phi}$. We refer the readers to two theses about semigroup and monoid presentations $[3,8]$.

\section{Preliminaries}

Let $\alpha \in \mathcal{T}_{n}$, then the kernel and image of $\alpha$ are defined by

$$
\begin{gathered}
\operatorname{ker}(\alpha)=\left\{(x, y) \in X_{n} \times X_{n} \mid x \alpha=y \alpha\right\} \\
\operatorname{im}(\alpha)=\left\{x \alpha \mid x \in X_{n}\right\} .
\end{gathered}
$$

Moreover, it is well known that if $\alpha, \beta \in \mathcal{T}_{n}$ then $\operatorname{im}(\alpha \beta) \subseteq \operatorname{im}(\beta)$ and $\operatorname{ker}(\alpha \beta) \supseteq \operatorname{ker}(\alpha)$.

Definition 2.1. Let $A$ be a non-empty subset of $X_{n}$. If $x, y \in A$ and $x \leq z \leq y \Longrightarrow z \in A$ for all $x, y \in A$, then $A$ is called a convex subset of $X_{n}$.

If $\alpha \in \mathcal{T}_{n}$ is a contraction mapping then $\operatorname{im}(\alpha)$ is a convex subset of $X_{n}$ [4]. Thus if $\alpha \in \mathcal{O} D C T_{n}$ then $\operatorname{im}(\alpha)$ is a convex subset of $X_{n}$. Moreover, from the definition of $\mathcal{O} D C T_{n}$ it is easy to see that if $\alpha \in \mathcal{O} D C T_{n}$ then $\operatorname{im}(\alpha)=\{1,2, \ldots, r\}$ for $1 \leq r \leq n$ and each equivalence kernel classes of $\alpha$ are convex subsets of $X_{n}$. Thus if $\alpha \in \mathcal{O} D C T_{n}$ then

$$
\alpha=\left(\begin{array}{cccc}
A_{1} & A_{2} & \ldots & A_{r} \\
1 & 2 & \ldots & r
\end{array}\right)
$$

for $1 \leq r \leq n$. Moreover, we have $x \geq i$ for $\forall x \in A_{i}$ and $\left\{A_{1}, A_{2}, \ldots A_{r}\right\}$ is a partition of $X_{n}$, if $a \in A_{i}$ and $b \in A_{j}$ for $1 \leq i<j \leq n$ then $a<b$. 


\section{The Monoid Rank of $O D C T_{n}$}

In this section, we have found a minimal generating set of $\mathcal{O D C T} T_{n}$ and we obtained the monoid rank of $\mathcal{O} D C T_{n}$. It is clear that $\left.\mathcal{O} D C T_{1}=\left\{\left(\begin{array}{l}1 \\ 1\end{array}\right)\right\}\right\}$ which is a clearly generated by empty set as a monoid and $\mathcal{O} D C T_{2}=$ $\left\{\left(\begin{array}{ll}1 & 2 \\ 1 & 1\end{array}\right),\left(\begin{array}{ll}1 & 2 \\ 1 & 2\end{array}\right)\right\}$ which is clearly generated by the element $\left(\begin{array}{ll}1 & 2 \\ 1 & 1\end{array}\right)$ as a monoid. Let $n \geq 3$ and $\mathcal{F}_{r}=$ $\left\{\alpha \in \mathcal{O} D C T_{n}:|\operatorname{im}(\alpha)|=r\right\}$ for $1 \leq r \leq n$. Notice that $\mathcal{F}_{n}=\left\{\epsilon=\left(\begin{array}{llll}1 & 2 & \ldots & n \\ 1 & 2 & \ldots & n\end{array}\right)\right\}$ where $\epsilon$ is the identity element of $\mathcal{O} D C T_{n}$.

Lemma 3.1. Let $n \geq 3$. If $\alpha \in \mathcal{F}_{r}$ then $\alpha \in<\mathcal{F}_{r+1}>$ for $1 \leq r \leq n-2$.

Proof. Let $n \geq 3$ and $\alpha \in \mathcal{F}_{r}$ for $1 \leq r \leq n-2$. Then we have

$$
\alpha=\left(\begin{array}{cccc}
A_{1} & A_{2} & \ldots & A_{r} \\
1 & 2 & \ldots & r
\end{array}\right)
$$

where $1 \leq r \leq n-2$, so there exists $i$ such that $\left|A_{i}\right| \geq 2$ for $1 \leq i \leq r$. Let $x_{i}$ be the maximum element in $A_{i}$. Let $\beta$ be a mapping such that

$$
\beta=\left(\begin{array}{cccccccc}
A_{1} & \ldots & A_{i-1} & A_{i} \backslash\left\{x_{i}\right\} & \left\{x_{i}\right\} & A_{i+1} & \ldots & A_{r} \\
1 & \ldots & i-1 & i & i+1 & i+2 & \ldots & r+1
\end{array}\right)
$$

for $i>1$ and

$$
\beta=\left(\begin{array}{ccccc}
A_{1} \backslash\left\{x_{1}\right\} & \left\{x_{1}\right\} & A_{2} & \ldots & A_{r} \\
1 & 2 & 3 & \ldots & r+1
\end{array}\right)
$$

for $i=1$. Then it is clear that $\beta \in \mathcal{F}_{r+1}$. Let $\gamma$ be the mapping defined as

$$
j \gamma=\left\{\begin{array}{ll}
j & \text { if } 1 \leq j \leq i \\
i & \text { if } j=i+1 \\
j-1 & \text { if } i+2 \leq j \leq r+1 \\
r+1 & \text { if } j>r+1
\end{array}\right\}
$$

then it is clear that $\gamma \in \mathcal{F}_{r+1}$ and $\alpha=\beta \gamma$, so $\alpha \in<\mathcal{F}_{r+1}>$.

Corollary 3.1. $\mathcal{F}_{r} \subseteq<\mathcal{F}_{r+1}>$ for each $1 \leq r \leq n-2$.

Corollary 3.2. Since $\mathcal{F}_{n}$ is the set that has only the identity mapping of $\mathcal{O} D C T_{n}$ then we have $<\mathcal{F}_{n-1}>=\mathcal{O} D C T_{n}$ for $n \geq 3$.

Corollary $3.3([1]) \cdot\left|\mathcal{F}_{r}\right|=\left(\begin{array}{c}n-1 \\ r-1\end{array}\right)$ for $1 \leq r \leq n$.

Corollary 3.4. $\operatorname{rank}_{M}\left(\mathcal{O} D C T_{n}\right) \leq n-1$ for $n \in \mathbb{Z}^{+}$since $\left|\mathcal{F}_{n-1}\right|=n-1$.

Corollary 3.5 ([1]). $\left|\mathcal{O} D C T_{n}\right|=2^{n-1}$ for $n \geq 1$.

Theorem 3.1. $\operatorname{rank}_{M}\left(\mathcal{O} D C T_{n}\right)=n-1$ for $n \in \mathbb{Z}^{+}$.

Proof. If $n=1$ or $n=2$ then result is clear, let $n \geq 3$. We have $\operatorname{rank}_{M}\left(\mathcal{O} D C T_{n}\right) \leq n-1$ from Corollary 3.4. Let

$$
\mathcal{O} D C T_{(n, r)}=\left\{\alpha \in \mathcal{O} D C T_{n}:|\operatorname{im}(\alpha)| \leq r\right\}
$$

for $1 \leq r \leq n-1$. It is clear that $\mathcal{O} D C T_{(n, r)}$ is an ideal of $\mathcal{O} D C T_{n}$. In particular, $\mathcal{O} D C T_{(n, n-2)}$ is an ideal of $\mathcal{O} D C T_{n}$. Moreover, there are $n-1$ different kernel classes in $\mathcal{F}_{n-1}$ and we have $\mathcal{F}_{n}=\{\epsilon\}$, so $\operatorname{rank}_{M}\left(\mathcal{O} D C T_{n}\right) \geq n-1$. Thus we have concluded that $\operatorname{rank}_{M}\left(\mathcal{O} D C T_{n}\right)=n-1$ for $n \in \mathbb{Z}^{+}$. 


\section{The Monoid Presentation of $O D C T_{n}$}

In this section, we have found a monoid presentation of $\mathcal{O} D C T_{n}$ for $n \geq 3$.

Proposition 4.1 ([8]). Let $A$ be a set and let $M$ be any monoid. Then any mapping $\phi: A \rightarrow M$ can be extended in a unique way to a homomorphism $\bar{\phi}: A^{*} \rightarrow M$.

Definition 4.1. Let $M$ be any monoid, let $B$ be a generating set of $M$, and let $\phi: A \rightarrow B$ be an onto mapping. By Proposition 4.1 the mapping $\phi$ can be extended in a unique way to an epimorphism $\bar{\phi}: A^{*} \rightarrow M$. Let $R \subseteq A^{*} \times A^{*}$ be a set of relations. The monoid $M$ is said to satisfy relations $R$ if for each $(u, v) \in R$ we have $u \bar{\phi}=v \bar{\phi}$.

Let $M$ be a finite monoid, $A \subseteq M$ and $<A>=M$. Let $R \subseteq A^{*} \times A^{*}$ be a set of relations, and let $W \subseteq A^{*}$. It is well known that if

(i) the generators $A$ of $M$ satisfy all the relations from $R$

(ii) for each word $w \in A^{*}$ there exists a word $\bar{w} \in W$ such that $w=\bar{w}$ is a consequence of $R$

(iii) $|W| \leq|M|$

then $<A \mid R>$ is a monoid presentation of $M$.

Let $n \geq 3$ and $\alpha_{i}$ be the mapping defined as

$$
\alpha_{i}=\left(\begin{array}{cccccccc}
1 & \ldots & i-1 & i & i+1 & i+2 & \ldots & n \\
1 & \ldots & i-1 & i & i & i+1 & \ldots & n-1
\end{array}\right)
$$

for $2 \leq i \leq n-1$ and

$$
\alpha_{1}=\left(\begin{array}{ccccc}
1 & 2 & 3 & \ldots & n \\
1 & 1 & 2 & \ldots & n-1
\end{array}\right)
$$

then it is clear that $\mathcal{F}_{n-1}=\left\{\alpha_{i} \mid 1 \leq i \leq n-1\right\}$.

Lemma 4.1. Let $n \geq 3$ and $\alpha_{i}$ be defined as above then $\alpha_{i} \alpha_{n-1}=\alpha_{i}$ for $1 \leq i \leq n-1$. In particular, $\left(\alpha_{n-1}\right)^{2}=\alpha_{n-1}$.

Proof. Let $n \geq 3$ and $\alpha_{i}$ be defined as above, then

$$
\alpha_{n-1}=\left(\begin{array}{ccccc}
1 & 2 & \ldots & n-1 & n \\
1 & 2 & \ldots & n-1 & n-1
\end{array}\right) .
$$

$1\left(\alpha_{i} \alpha_{n-1}\right)=1$ and $n\left(\alpha_{i} \alpha_{n-1}\right)=n-1$, we have $\operatorname{im}\left(\alpha_{i} \alpha_{n-1}\right)=\{1,2, \ldots, n-1\}$ from the definition of $\mathcal{O} D C T_{n}$. Moreover, $i\left(\alpha_{i} \alpha_{n-1}\right)=i$ and $(i+1)\left(\alpha_{i} \alpha_{n-1}\right)=i$, so $\alpha_{i} \alpha_{n-1}=\alpha_{i}$ for $1 \leq i \leq n-1$.

Lemma 4.2. Let $n \geq 3$ and $\alpha_{i}$ be defined as above then $\alpha_{i} \alpha_{j}=\alpha_{j+1} \alpha_{i}$ for $1 \leq i \leq j \leq n-2$.

Proof. Let $n \geq 3, \alpha_{i}$ be defined as above and $1 \leq i \leq j \leq n-2$. It is clear that $1\left(\alpha_{i} \alpha_{j}\right)=1$ and $n\left(\alpha_{i} \alpha_{j}\right)=n-1\left(\alpha_{j}\right)=$ $n-2$ since $1 \leq i \leq j \leq n-2$. Thus $\operatorname{im}\left(\alpha_{i} \alpha_{j}\right)=\{1,2, \ldots, n-2\}$ from the definition of $\mathcal{O} D C T_{n}$. Moreover we have

$$
\begin{gathered}
i\left(\alpha_{i} \alpha_{j}\right)=i \alpha_{j}=i \\
(i+1)\left(\alpha_{i} \alpha_{j}\right)=i \alpha_{j}=i \\
(j+1)\left(\alpha_{i} \alpha_{j}\right)=j \alpha_{j}=j \\
(j+2)\left(\alpha_{i} \alpha_{j}\right)=(j+1) \alpha_{j}=j .
\end{gathered}
$$

Also, $1\left(\alpha_{j+1} \alpha_{i}\right)=1$ and $n\left(\alpha_{j+1} \alpha_{i}\right)=(n-1) \alpha_{i}=n-2$ thus $\operatorname{im}\left(\alpha_{j+1} \alpha_{i}\right)=\{1,2, \ldots, n-2\}$ from the definition of $\mathcal{O} D C T_{n}$. Moreover we have

$$
\begin{aligned}
i\left(\alpha_{j+1} \alpha_{i}\right) & =i \alpha_{i}=i \\
(i+1)\left(\alpha_{j+1} \alpha_{i}\right) & =(i+1) \alpha_{i}=i \\
(j+1)\left(\alpha_{j+1} \alpha_{i}\right) & =(j+1) \alpha_{i}=j \\
(j+2)\left(\alpha_{j+1} \alpha_{i}\right) & =(j+1) \alpha_{i}=j .
\end{aligned}
$$

Therefore, $x\left(\alpha_{i} \alpha_{j}\right)=x\left(\alpha_{j+1} \alpha_{i}\right)$ for $\forall x \in X_{n}$. It follows that $\alpha_{i} \alpha_{j}=\alpha_{j+1} \alpha_{i}$ for $1 \leq i \leq j \leq n-2$. 
Definition 4.2. Let $A$ be a finite set and $w=a_{1} a_{2} \ldots a_{k}$ for $a_{i} \in A$ and $1 \leq i \leq k$. Length of $w$ is defined as $k$ and we write $l(w)=k$ and if $w$ is empty word then the length of $w$ is defined as 0 (zero) and we write $l(w)=0$.

Theorem 4.1. Let $n \geq 3$. Let $A=\left\{a_{1}, a_{2}, \ldots, a_{n-1}\right\}$ and $R=\left\{a_{i} a_{n-1}=a_{i}(1 \leq i \leq n-1), a_{i} a_{j}=a_{j+1} a_{i}(1 \leq i \leq\right.$ $j \leq n-2)\}$. Then $<A \mid R>$ is a monoid presentation of $\mathcal{O} D C T_{n}$ for $n \geq 3$.

Proof. Let $n \geq 3$. Let $A=\left\{a_{1}, a_{2}, \ldots, a_{n-1}\right\}$ and $R=\left\{a_{i} a_{n-1}=a_{i}(1 \leq i \leq n-1), a_{i} a_{j}=a_{j+1} a_{i}(1 \leq i \leq j \leq\right.$ $n-2)\}$. Let $f: A \rightarrow \mathcal{F}_{n-1}$ be the mapping such that $a_{i} f=\alpha_{i}$. There exists a unique epimorpish $\bar{f}: A^{*} \rightarrow \mathcal{O} D C T_{n}$ extending the $f$. Thus $\mathcal{O} D C T_{n}$ satisfies all the relations from $R$ since Lemma 4.1 and Lemma 4.2. Let $\varepsilon$ is the empty word and

$$
W=\left\{a_{j_{k}} a_{j_{k-1}} \ldots a_{j_{1}} \mid n-1 \geq j_{k}>j_{k-1}>\ldots>j_{1} \geq 1\right\} \cup\{\varepsilon\} .
$$

Thus it is clear that $W \subseteq A^{*}$ and $|W|=2^{n-1}$. Let $w \in A^{*}$ and $l(w)=m$. We will show that there exists $\bar{w} \in W$ such that $w=\bar{w}$ is a consequence of $R$. We use induction on $m$. If $m=0$ or $m=1$, then the result is clear. Let $m \geq 2$, then $w \equiv w_{1} w_{2}$ where $l\left(w_{1}\right)=m-1$ and $l\left(w_{2}\right)=1$. Thus $w_{2} \in A$, moreover we have $w_{1}=\overline{w_{1}}$ such that $\overline{w_{1}} \in W$ from the induction hypothesis. So $w=\overline{w_{1}} w_{2}$. If $\overline{w_{1}} \equiv \varepsilon$ then result is clear. Let $\overline{w_{1}} \not \equiv \varepsilon$. Then,

$$
\overline{w_{1}} \equiv a_{t_{p}} a_{t_{p-1}} \ldots a_{t_{1}}
$$

where $n-1 \geq t_{p}>t_{p-1}>\ldots>t_{1} \geq 1$ and $w=a_{t_{p}} a_{t_{p-1}} \ldots a_{t_{1}} w_{2}$. If $w_{2} \equiv a_{n-1}$ then

$$
\begin{gathered}
w=a_{t_{p}} a_{t_{p-1}} \ldots a_{t_{1}} a_{n-1} \\
w=a_{t_{p}} a_{t_{p-1}} \ldots a_{t_{1}}
\end{gathered}
$$

so in this case $w=\overline{w_{1}}$ and $\overline{w_{1}} \in W$. Let $w_{2} \equiv a_{i}$ where $1 \leq i \leq n-2$. Then

$$
w=a_{t_{p}} a_{t_{p-1}} \ldots a_{t_{1}} a_{i}
$$

if $t_{1}>i$ then we have $\bar{w} \equiv a_{t_{p}} a_{t_{p-1}} \ldots a_{t_{1}} a_{i}$ and $w=\bar{w}, \bar{w} \in W$. If $t_{1} \leq i$ then

$$
\begin{gathered}
w=a_{t_{p}} a_{t_{p-1}} \ldots a_{t_{2}} a_{t_{1}} a_{i} \\
w=a_{t_{p}} a_{t_{p-1}} \ldots a_{t_{2}} a_{i+1} a_{t_{1}} .
\end{gathered}
$$

If $p=1$ then result is clear, let $p \geq 2$. If $t_{2}>i+1$ then we have $\bar{w} \equiv a_{t_{p}} a_{t_{p-1}} \ldots a_{t_{2}} a_{i+1} a_{t_{1}}$ and $w=\bar{w}, \bar{w} \in W$. If $i+1=n-1$ then

$$
\begin{gathered}
w=a_{t_{p}} a_{t_{p-1}} \ldots a_{t_{2}} a_{i+1} a_{t_{1}} \\
w=a_{t_{p}} a_{t_{p-1}} \ldots a_{t_{2}} a_{n-1} a_{t_{1}} \\
w=a_{t_{p}} a_{t_{p-1}} \ldots a_{t_{2}} a_{t_{1}},
\end{gathered}
$$

so in this case $\bar{w} \equiv a_{t_{p}} a_{t_{p-1}} \ldots a_{t_{2}} a_{t_{1}}$ and $w=\bar{w}, \bar{w} \in W$. If $t_{2} \leq i+1<n-1$ then we have

$$
\begin{aligned}
& w=a_{t_{p}} a_{t_{p-1}} \ldots a_{t_{2}} a_{i+1} a_{t_{1}} \\
& w=a_{t_{p}} a_{t_{p-1}} \ldots a_{i+2} a_{t_{2}} a_{t_{1}} .
\end{aligned}
$$

If we use the same algorithm, it is clear that finally we conclude that there exists a word $\bar{w} \in W$ such that $w=\bar{w}$ is a consequence of $R$. Moreover, $|W|=\left|\mathcal{O} D C T_{n}\right|=2^{n-1}$, it follows that $\langle A \mid R\rangle$ is a monoid presentation of $\mathcal{O} D C T_{n}$ for $n \geq 3$.

\section{Conclusion}

In this paper we have found monoid rank of $\mathcal{O} D C T_{n}$ for $n \in \mathbb{Z}^{+}$. Moreover since $\mathcal{O} D C T_{1}$ is a trivial monoid and $\mathcal{O} D C T_{2}$ is a monogenic monoid, we give a monoid presentation of $\mathcal{O} D C T_{n}$ for $n \geq 3$. Recently, the rank of $\mathcal{O} C T_{n}$ and the rank of $\mathcal{O} R C T_{n}$ have been found, finding presentation problem can be considered on those semigroups as a future work.

\section{Funding}

There is no funding for this work. 


\section{Availability of data and materials}

Not applicable.

\section{Competing interests}

The authors declare that they have no competing interests.

\section{Author's contributions}

All authors contributed equally to the writing of this paper. All authors read and approved the final manuscript.

\section{References}

[1] Adeshola, A.D., Umar, A.: Combinatorial results for certain semigroups of order-preserving full contraction mappings of a finite chain. Journal of Combinatorial Mathematics and Combinatorial Computing. 106, 37-49 (2018).

[2] Ayık, G., Ayık H., Koç, M.: Combinatorial results for order-preserving and order-decreasing transformations. Turkish Journal of Mathematics. 35 (4), 617-625 (2011).

[3] Ayık, H.: Presentations and efficiency of semigroups. Ph. D. Thesis. Universtiy of St Andrews (1998).

[4] Garba, G.U., Ibrahim, M.J., Imam, A.T.: On certain semigroups of full contraction maps of a finite chain. Turkish Journal of Mathematics. 41 (3), 500-507 (2017).

[5] Gomes, M.S., Howie, J.M.: On the ranks of certain semigroups of order-preserving transformations. Semigroup Forum. 45 (1), 272-282 (1992).

[6] Higgins, P.M.: Combinatorial results for semigroups of order-preserving mappings. Mathematical Proceedings of the Cambridge Philosophical Society. 113 (2), 281-296 (1993).

[7] Howie, J.M.: Fundamentals of semigroup theory. Oxford University Press. New York (1995).

[8] Ruskuc, N.: Semigroup presentations. Ph. D. Thesis. Universtiy of St Andrews (1995).

\section{Affiliations}

KEMAL TOKER

AdDRESS: Harran University, Department of Mathematics, Faculty of Science and Literature, 63000, Şanllurfa Turkey.

E-MAIL: ktoker@harran.edu.tr

ORCID ID: 0000-0003-3696-1324 